\title{
Phase structure of four-dimensional gonihedric spin system
}

\author{
G.Koutsoumbas \\ Physics Department, National Technical University \\ Zografou Campus, 15780 Athens, Greece \\ G.K.Savvidy \\ National Research Center "Demokritos", \\ Ag. Paraskevi, GR-15310 Athens, Greece \\ K.G.Savvidy \\ Princeton University, Department of Physics \\ P.O.BOX 708, Princeton, New Jersey 08544, USA
}

\begin{abstract}
We perform Monte Carlo simulations of a gauge invariant spin system which describes random surfaces with gonihedric action in four dimensions. The Hamiltonian is a mixture of one-plaquette and additional two- and threeplaquette interaction terms with specially adjusted coupling constants [1, 2]. For the system with the large self-intersection coupling constant $k$ we observe the second-order phase transition at temperature $\beta_{c} \simeq 1.75$. The string tension is generated by quantum fluctuations as it was expected theoretically [9]. This result suggests the existence of a noncritical string in four dimensions. For smaller values of $k$ the system undergoes the first order phase transition and for $k$ close to zero exhibits a smooth crossover.
\end{abstract}




\section{Introduction}

The gonihedric string has been defined as a model of random surfaces with an action which is proportional to the linear size of the surface $[7,8,9]$

$$
A(M)=\sum_{<i j>} \lambda_{i j} \cdot \Theta\left(\alpha_{i j}\right), \quad \Theta(\alpha)=|\pi-\alpha|^{\varsigma},
$$

where $\lambda_{i j}$ is the length of the edge $\left\langle i j>\right.$ of the triangulated surface $M_{2}$ and $\alpha_{i j}$ is the dihedral angle between two neighbouring triangles of $M$ sharing a common edge $<i j\rangle$. The angular factor $\Theta$ defines the rigidity of random surfaces and for $\varsigma \leq 1$ the angular factor increases sufficiently fast near angles $\alpha=\pi$ to suppress transverse fluctuations 9]. The gonihedric action has been defined for self-intersecting surfaces as well 9]. The action accounts self-intersections of different orders by ascribing weights to self-intersections. These weights are proportional to the length of the intersection multiplied by the angular factor which is equal to the sum of all angular factors corresponding to dihedral angles in the intersection [9]. The coupling constant in front of this term is called self-intersection coupling constant [2]. In principle this coupling constant is a free parameter of the theory, but if one applies the continuity principle then one can fix the value of $k_{c}=1 / 2$ [9, 2] (see formulas (4) and (28) in [2]).

The model has a number of properties which bring it very close to the Feynman path integral for a point-like relativistic particle [8, 9]. This can be seen from (1) in the limit when the surface degenerates into a single world line, and in that case the action is proportional to the length of the path. This property of the gonihedric action guarantees that the spike instability, which is common to other triangulated random surface theories, does not appear here, because the action is proportional to the total length of the spikes and thus suppresses the corresponding fluctuations [0, 8, 9].

The other important property of the theory is that at the classical level the string tension is equal to zero and quarks viewed as open ends of the surface are propagating freely without interaction [9]. This is because the gonihedric action (11) is equal to the perimeter of the flat Wilson loop [9]. As it was demonstrated in [9], quantum fluctuations generate a nonzero string tension

$$
\sigma_{\text {quantum }}=\frac{d}{a^{2}}\left(1-\ln \frac{d}{\beta}\right)
$$

where $d$ is the dimension of the spacetime, $\beta$ is the coupling constant, $a$ is a scaling parameter and

$$
\varsigma=\frac{d-2}{d}
$$

in (1). In the scaling limit $\beta \rightarrow \beta_{c}=d / e$ the string tension has a finite limit while the scaling parameter tends to zero as

$$
a=\left(\beta-\beta_{c}\right)^{1 / 2}
$$


thus the critical exponent $\nu$ is equal to one half [9]

$$
\nu=1 / d_{H}=1 / 2
$$

where $d_{H}$ is the Hausdorff dimension. Thus, although at the tree level the theory describes free quarks with string tension equal to zero, quantum fluctuations generate nonzero string tension and, as a result, the quark confinement [9]. The gonihedric string may consistently describe asymptotic freedom and confinement, as it is expected to be the case in QCD円.

In addition to the formulation of the theory in the continium space $R^{d}$ the system allows an equivalent representation on Euclidean lattice $Z^{d}$ where a surface is associated with a collection of plaquettes. Lattice spin systems whose interface energy coinsides with the action (1) have been constructed in arbitrary dimension $d$ [1] for the self-intersection coupling constant $k=1$ and for an arbitrary $k$ in [2]. This gives an opportunity for numerical simulations of the corresponding statistical systems in a way which is similar to the Monte Carlo simulations of QCD [13].

In three dimensions the corresponding Hamiltonian is equal to [1, 2]

$$
H_{\text {gonihedric }}^{3 d}=-2 k \sum_{\vec{r}, \vec{\alpha}} \sigma_{\vec{r}} \sigma_{\vec{r}+\vec{\alpha}}+\frac{k}{2} \sum_{\vec{r}, \vec{\alpha}, \vec{\beta}} \sigma_{\vec{r}} \sigma_{\vec{r}+\vec{\alpha}+\vec{\beta}}-\frac{1-k}{2} \sum_{\vec{r}, \vec{\alpha}, \vec{\beta}} \sigma_{\vec{r}} \sigma_{\vec{r}+\vec{\alpha}} \sigma_{\vec{r}+\vec{\alpha}+\vec{\beta}} \sigma_{\vec{r}+\vec{\beta}}
$$

and is a natural extension of the $3 D$ Ising model

$$
H_{\text {Ising }}^{3 d}=-\sum_{\vec{r}, \vec{\alpha}} \sigma_{\vec{r}} \sigma_{\vec{r}+\vec{\alpha}}
$$

to the gonihedric case. The extensions of the $3 D$ Ising model have been considered in the literature [5] and their phase structure has been investigated. The essential point is that the geometrical nature of the gonihedric system specifies the coupling constants and the symmetry of the system?2 This is in analogy [1, 2] with supersymmetric systems where the symmetry also specifies the coupling constants.

The self-intersection coupling constant $k$ defines the degree of degeneracy of the vacuum state [16, 15]; if $k \neq 0$, the degeneracy of the vacuum state is equal to $3 \cdot 2^{N}$ for the lattice of the size $N^{3}$ and is equal to $2^{3 N}$ when $k=0$. The last case is a sort of supersymmetric point in the space of gonihedric Hamiltonians. What is amazing is that not only the ground state, but all of the energy levels have the same exponential degeneracy. This enhanced symmetry at the point $k=0$ allows to construct the dual Hamiltonian in three dimensions [15, 16]. For $k \neq 0$ the degeneracy of energy levels is smaller compared with the vacuum state. This exponential degeneracy is reminiscent of spin glass systems.

\footnotetext{
1 Different modifications of the action (11) have been proposed in the literature by adding the area term or the gaussian term. In the first case this will cause a nonzero string tension already at the classical level and will bring back all problems of the critical string when applied to strong interactions. In the second case the model becomes simply equivalent to the gaussian model [8] (see formulas (46)-(48)).

${ }^{2}$ Spoiling of this fine tuning of coupling constants is equivalent to an addition of an area term and thus leads to nonzero string tension at the tree level (see previous footnote) and "drives" the system to an unwanted class of universality.
} 
Despite the fact that the system has a rich symmetry, in three dimensions the two-point correlation function remains as a fundamental observable [17]

$$
C(\vec{r})=<\sigma_{\vec{o}} \sigma_{\vec{r}}>
$$

and its behaviour at large distances is the best indicator of the phase transitions in the 3D gonihedric spin system. As usual, the most direct indication of the secondorder phase transition is the growth of the correlation length, which in the scaling limit should tend to infinity. The singularities of the energy, of the specific heat and of the generalized magnetization are also indicative of the order of the phase transition. The generalized magnetization is defined as the vacuum expectation value of the projection of spin states to the one which corresponds to the vacuum spin configuration [17

$$
M^{\mu}=<\sum_{\vec{r}} \sigma_{\vec{r}}^{\mu}(v a c) \cdot \sigma_{\vec{r}}>
$$

where $\sigma_{\vec{r}}^{\mu}(v a c)$ denotes the vacuum spin configurations and $\mu=1,2, \ldots, 2^{3 N}$ for the case $k=0$ and $\mu=1,2, \ldots, 3 \cdot 2^{N}$ in all other cases. The exponential degeneracy of the ground state is common to gonihedric spin systems and the analogy with spin glasses allows to construct the generalized magnetization (5) [17. In the real experiments one can choose some subset of generalized magnetizations (5) and measure the projection of virtual states to that subset of order parameters [17].

The first Monte Carlo simulations [17] demonstrated that the gonihedric system with intersection coupling constant equal to one, $k=1$, undergoes the secondorder phase transition at $\beta_{c} \approx 0.44$. This happens near the critical temperature of the two-dimensional Ising model $\beta_{c}=\frac{1}{2} \ln (1+\sqrt{2}) \approx 0.44$ [15]. This result follows from the transfer matrix approach [15] which describes the propagation of the two-dimensional system with the length and curvature amplitude. The system has a continuum limit at this temperature and describes two-dimensional free Dirac fermion [15]. At the same time it was not expected that the critical indices will coincide with the ones of the 2D Ising model simply because the physical picture of the fermionic string propagation which follows from the transfer matrix approach [15] is different from the propagation of the free point like fermion in two dimensions. In addition the analytical result (3) predicts the value $\nu=0.5$, which does not coinside with the critical exponent of the $2 \mathrm{D}$ Ising model $\nu=1$. The Monte Carlo simulations 17] confirm that the value of $\nu$ is small, $\nu \approx 0.34$, and is almost twice smaller than that for the 3D Ising model where $\nu=0.63$ [21]. In [19] the value $\nu \approx 0.44$ is obtained. Thus the correlation function grows slower near the critical point. This means that the 3D gonihedric system and the 3D Ising model are in different classes of universality.

Our aim is to study the phase structure of the spin systems which simulate random surfaces with gonihedric action in four dimensions. As it is well known, random surfaces with an area action in four dimensions can be simulated by the oneplaquette gauge invariant action (7) [3]. It should be reminded also that this gauge invariant spin system in four dimensions is self-dual and the critical temperature is equal to $\beta_{c}=\frac{1}{2} \ln (1+\sqrt{2})$ [3]. The Monte Carlo simulation of the system strongly 
indicates that the phase transition in $4 \mathrm{D} Z_{2}$ gauge invariant spin system is of the first order [4, 14.

In the next section we shall describe the corresponding gonihedric Hamiltonian from [1, 2] and the appropriate observables. The results of the Monte Carlo simulations are presented in the fourth section. For the system with the self-intersection coupling constant $k>k_{c}^{\prime}=1 / 2$ we observe the second-order phase transition at temperature $\beta_{c} \simeq 1.75$. The string tension is generated by quantum fluctuations as it was expected theoretically [9]. This result suggests the existance of a noncritical string theory in four dimensions.

For smaller values of $k$ the system undergoes a first-order phase transition at temprature $\beta_{c} \geq 2$. The passage from second-order phase transition to the first-order one is caused by the condensation of self-intersections. Finally, for $k<k_{c}^{\prime \prime}=1 / 6$ the system exhibits a smooth crossover. Thus two critical values of $k$ separate three regions of different critical behaviour and demonstrate the essential dependence of the phase structure on the intersection coupling constant $k$.

\section{Gonihedric spin system in four dimensions}

The system of random surfaces with gonihedric action can be formulated not only in three dimensions, but also in any dimension [1]. Similar to the three-dimensional case, the surface can be associated with a collection of plaquettes on a lattice and the interaction between spins can be organized in a way that the surfaces of interface will have the gonihedric action [四].

In four dimensions the gonihedric Hamiltonian essentially differs from the threedimensional one (1) because now it represents a gauge invariant spin system. The gauge invariant Hamiltonian in four dimensions has the form [1]

$$
H_{\text {gonihedric }}^{4 d}=-\frac{4}{g^{2}} \sum_{\{\text {plaquettes }\}}(\sigma \sigma \sigma \sigma)+\frac{1}{4 g^{2}} \sum_{\{\text {right angle plaquettes }\}}\left(\sigma \sigma \sigma \sigma_{\alpha}\right)^{r t}\left(\sigma_{\alpha} \sigma \sigma \sigma\right),
$$

where $g^{2}$ is the gauge coupling constant and the independent spin variables $\sigma_{i j}$ should be attached to the centers of the edges $\langle i, j\rangle$ of the four-dimensional lattice.

The Hamiltonian which simulates random surfaces with area action in four dimensions is well known [3] and represents a gauge invariant spin system with oneplaquette interaction term

$$
H_{\text {area }}^{4 d}=-\frac{1}{g^{2}} \sum_{\{\text {plaquettes }\}}(\sigma \sigma \sigma \sigma) .
$$

Thus the gonihedric Hamiltonian (6) in addition to the "ferromagnetic" interaction (7) on each elementary plaquette has the "antiferromagnetic" interaction between two plaquettes which form a right angle [1]

$$
J_{\text {plaquettes }}=-16 \cdot J_{r t \text { plaquettes }}=\frac{4}{g^{2}} \text {. }
$$

The partition function corresponding to (6) is equal to 


$$
Z(\beta)=\sum_{\left\{\sigma_{\vec{r}}\right\}} e^{-\beta g^{2} H / 4}
$$

These formulas completely define the gonihedric system in four dimensions and allow to simulate random surfaces on a four-dimensional lattice. It is important to stress that both systems (6) and (7) are of a geometrical nature because in the first case the amplitudes are proportional to the linear size of a surface and in the second case they are proportional to the area. The other difference between these two systems is that in the gonihedric case (6) the self-intersections of the surface can be propertly counted [9, 2]. There are various formulations of self-avoiding random surfaces on a lattice [6]. The main difference between these models and the gonihedric case is that the energy ascribed to self-intersections essentially depends on the geometry of intersecting plaquettes.

Indeed, the essential property of the spin realization of the gonihedric system (6) is that it properly counts the self-intersections [9]. On a four-dimensional lattice a two-dimensional closed surface can have self-intersections of different orders because at a given edge one can have intersections of four or six plaquettes [1]. The energy ascribed to a self-intersection essentially depends on a configuration of plaquettes in the intersection [9], and there are two topologically different configurations of plaquettes with four intersecting plaquettes and only one with six intersecting plaquettes. The corresponding energies as they are defined by the Hamiltonian (6) $\left(g^{2}=1\right)$ are equal to [1]

$$
\epsilon_{2}=1, \quad \bar{\epsilon}_{4}=4, \quad \overline{\bar{\epsilon}}_{4}=5, \quad \epsilon_{6}=12
$$

and are equal to the number of plaquettes which intersect at a right angle. The total energy of the surface which has $n_{2}$ edges with two intersecting plaquettes at a right angle - "simple edges" $3, \bar{n}_{4}$ and $\bar{n}_{4}$ edges with intersection of four plaquettes and $n_{6}$ with six plaquettes is equal to

$$
\epsilon=n_{2}+4 \bar{n}_{4}+5 \overline{\bar{n}}_{4}+12 n_{6}
$$

In the article [2] the intersection coupling constant $k$ was introduced to control the intensity of self-intersections. In that case the Hamiltonian has the form [2]

$$
\begin{aligned}
H_{\text {gonihedric }}^{\kappa} & =-\frac{5 \kappa-1}{g^{2}} \sum_{\{\text {plaquettes }\}}(\sigma \sigma \sigma \sigma)+\frac{\kappa}{4 g^{2}} \sum_{\{\text {right angle plaquettes }\}}\left(\sigma \sigma \sigma \sigma_{\alpha}\right)^{r t}\left(\sigma_{\alpha} \sigma \sigma \sigma\right) \\
& -\frac{1-\kappa}{8 g^{2}} \quad \sum_{\{\text {triples of right angle plaquettes }\}}\left(\sigma \sigma \sigma \sigma_{\alpha}\right)^{r t}\left(\sigma_{\alpha} \sigma \sigma \sigma_{\beta}\right)^{r t}\left(\sigma_{\beta} \sigma \sigma \sigma\right) .
\end{aligned}
$$

As it can be seen from the last expression in contrast to the case $\kappa=1$ (ब6) there appears a new three-plaquette interaction term?.

The corresponding energies ascribed to self-intersections are equal to [2]

\footnotetext{
${ }^{3}$ edges with two intersecting plaquettes at a right angle

${ }^{4}$ The coefficient in front of this term is twice smaller than that in 2 because for convinience we take all symmetric combinations of the plaquette triples inside the $3 \mathrm{~d}$ cube.
} 


$$
\epsilon_{2}=1, \quad \bar{\epsilon}_{4}=4 k, \quad \overline{\bar{\epsilon}}_{4}=6 k-1, \quad \epsilon_{6}=12 k
$$

and coinside with the weights (8) when $k=1$. The total energy of the surface in this general case is

$$
\epsilon=n_{2}+4 k \bar{n}_{4}+(6 k-1) \overline{\bar{n}}_{4}+12 k n_{6}
$$

and reduces to (9) when $k=1$.

Unfortunately the method used for the construction of the transfer matrix in three dimensions [9] can not be used in four dimensions and we face an even a more complicated problem.

\section{Observables}

In pure gauge theories the magnetization is always equal to zero, $\langle\sigma\rangle=0$, therefore this quantity can not be the order parameter. In other words the system does not have local order parameters to distinguish the phases and only gauge invariant products of spin variables over closed loops can be nonzero

$$
W(C)=<\prod_{C} \sigma>
$$

and, as it was pointed out by Wegner [3], can separate the phases of the system. The perimeter and the area law of (12) can distinguish the ordered and disordered phases [3] (see formulas (3.34) - (3.39) in [3]). The same conclusion is correct for the gonihedric systems (6) and ([10), therefore only different loop products are to be considered.

To define the phase structure of the four-dimensional gonihedric gauge system one can measure different observables. The simplest one is the total energy per edge which can be expressed as a combination of different loop products of spins inside a three-dimensional cube [2]

$$
\begin{aligned}
H_{\text {edge }}^{\text {tot }}=3 k & -\frac{5 \kappa-1}{4} \sum_{\{\text {six }\}}(\sigma \sigma \sigma \sigma)+\frac{\kappa}{4} \sum_{\{\text {twelve }\}}\left(\sigma \sigma \sigma \sigma_{\alpha}\right)^{r t}\left(\sigma_{\alpha} \sigma \sigma \sigma\right) \\
& -\frac{1-\kappa}{8} \sum_{\{\text {twelve }\}}\left(\sigma \sigma \sigma \sigma_{\alpha}\right)^{r t}\left(\sigma_{\alpha} \sigma \sigma \sigma_{\beta}\right)^{r t}\left(\sigma_{\beta} \sigma \sigma \sigma\right) .
\end{aligned}
$$

Here the first term denotes the product of spins around one plaquette in a $3 \mathrm{~d}$ cube, the second one - a product of spins around two perpendicular plaquettes and the last one - a product of spins around three perpendicular plaquettes inside a $3 \mathrm{~d}$ cube of the four-dimensional lattice.

The advantage of this formulation of the theory is that one can measure the part of the surface energy which corresponds only to a simple edge which is proportional to $n_{2}$

$$
16 H_{\text {edge }}^{\text {simple }}=6+\sum_{\{\text {six }\}}(\sigma \sigma \sigma \sigma)-2 \sum_{\{\text {three }\}}(\sigma \sigma \sigma \sigma)^{\|}(\sigma \sigma \sigma \sigma)
$$




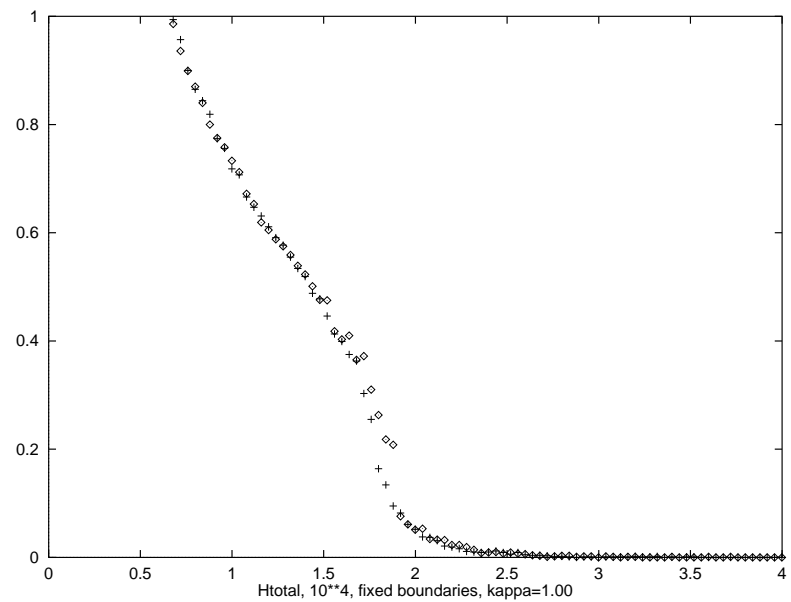

Figure 1: The average energy per edge (13) as a function of $\beta$. The system was heated and then cooled on steps of 0.001 , points are plotted every fortieth step, $k=1$.

$$
-\sum_{\{t w e l v e\}}\left(\sigma \sigma \sigma \sigma_{\alpha}\right)^{r t}\left(\sigma_{\alpha} \sigma \sigma \sigma_{\beta}\right)^{r t}\left(\sigma_{\beta} \sigma \sigma \sigma\right) .
$$

The separation of the simple edge energy allows to compute the part of the surface energy which corresponds to self-intersections

$$
H_{\text {edge }}^{\text {self-intersections }}=H_{\text {edge }}^{\text {tot }}-H_{\text {edge }}^{\text {simple }}
$$

One can express this observables directly in terms of numbers of intersections

$$
\begin{gathered}
H_{\text {edge }}^{\text {tot }} \approx n_{2}+4 k \bar{n}_{4}+(6 k-1) \overline{\bar{n}}_{4}+12 k n_{6} \\
H_{\text {edge }}^{\text {simple }} \approx n_{2} \\
H_{\text {edge }}^{\text {self-intersections }} \approx 4 k \bar{n}_{4}+(6 k-1) \overline{\bar{n}}_{4}+12 k n_{6}
\end{gathered}
$$

The other important observable is the surface area which can be computed by using one-plaquette product

$$
\Sigma_{\text {edge }}=3-\frac{1}{2} \sum_{\{\text {six }\}}(\sigma \sigma \sigma \sigma),
$$

where $(\sigma \sigma \sigma \sigma)=P$. The advantage to work with these observables is that they allow to control the inportance of the self-intersections in our attempt to find a nontrivial theory in four dimensions.

\section{Monte Carlo simulations}

As it is well known, random surfaces with an area action can be simulated by the gauge invariant action (17) [3]. It should be reminded that this gauge invariant spin system in four dimensions is self-dual and the critical temperature is equal to $\beta_{c}=$ $\frac{1}{2} \ln (1+\sqrt{2})$ [3]. The Monte Carlo simulation of the system strongly indicates that the phase transition in $4 \mathrm{D} Z_{2}$ gauge invariant spin system is of the first order [4, 14]. 


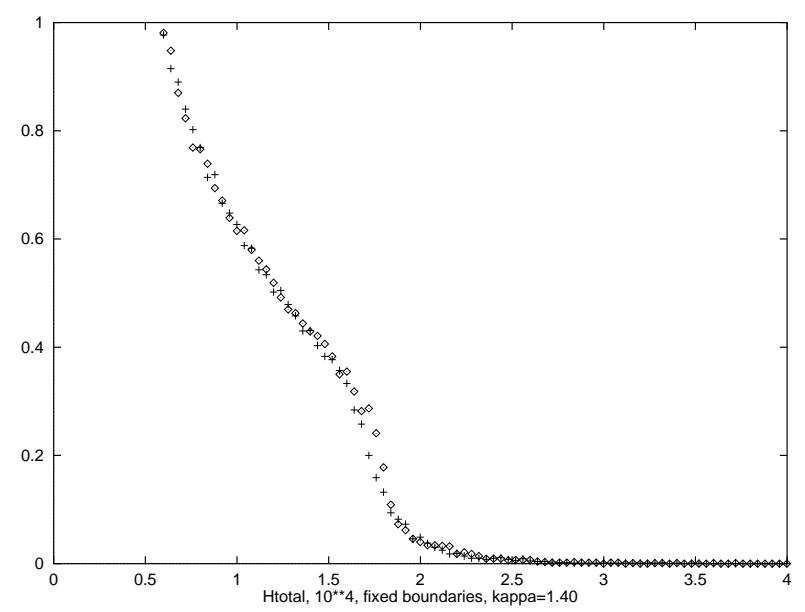

Figure 2: Total energy on edge (13), $k=1.4$

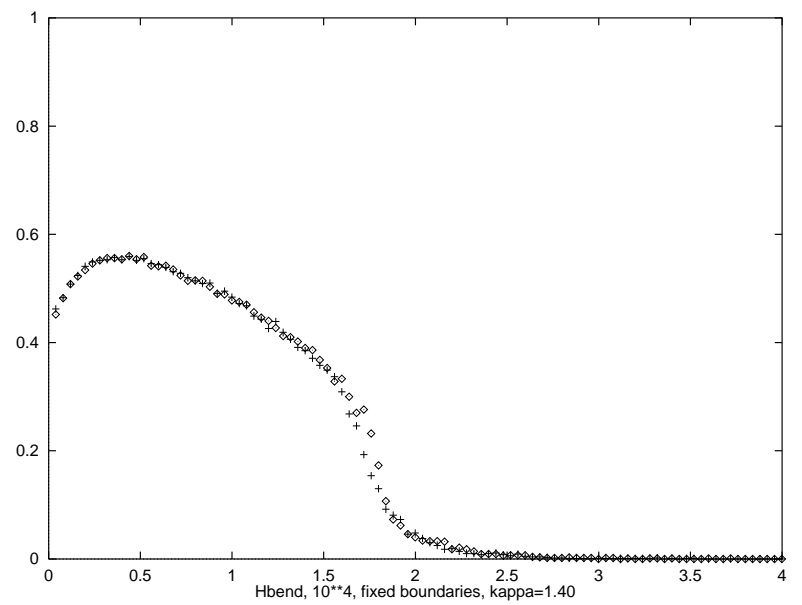

Figure 3: Simple edges (14), $k=1.4$

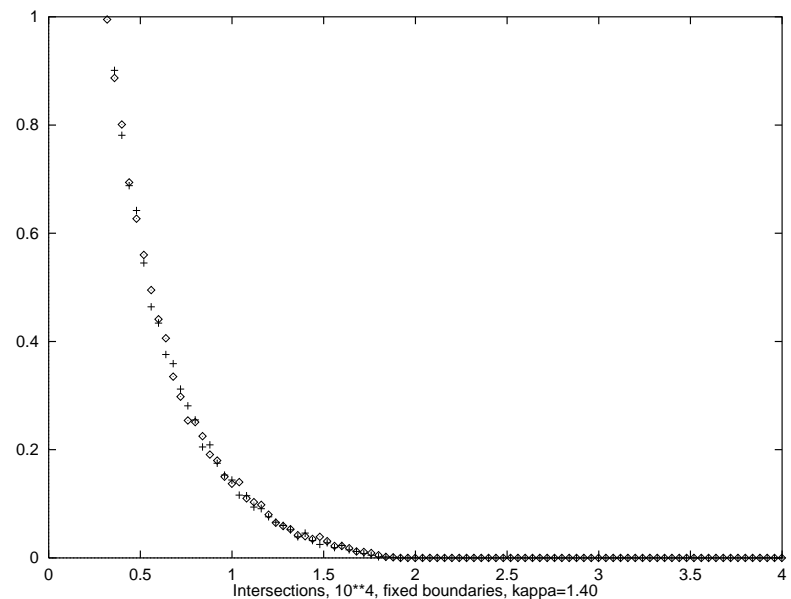

Figure 4: Self-intersection edges, $k=1.4$ 


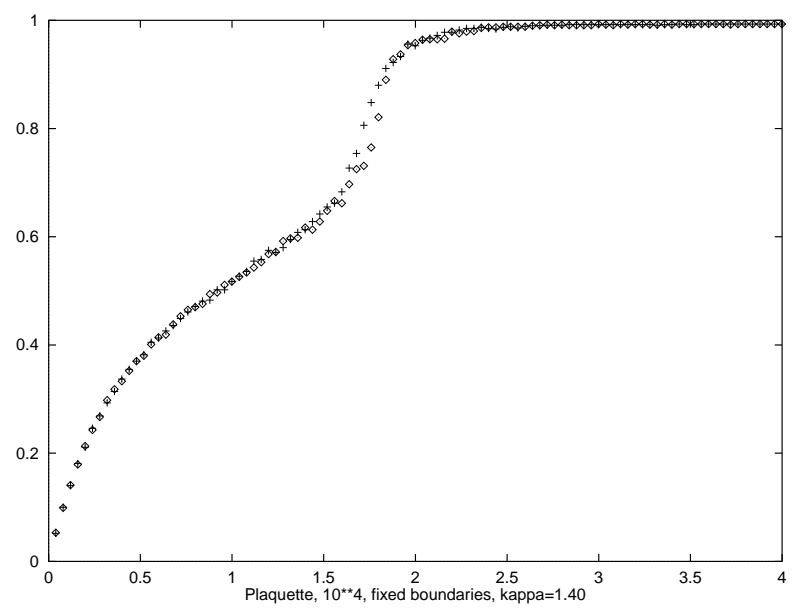

Figure 5: Plaquette, $k=1.4$

This has been indicated by measuring one-plaquette average (15) in the thermal cycle. A thermal cycle of the statistical system provides a general overview of its phase structure and is very helpful in defining the regions of the phase transitions [⿴囗十). Clear hysteresis in 4D $Z_{2}$ gauge invariant spin system is strongly indicative of the first-order phase transition [4]. The hysteresis is due to the metastability of the ordered phase at high temperature and of the disordered phase at low temperature.

The similar Monte Carlo simulations of the gonihedric system (6) and (10) on the lattices of the sizes $4^{4}, 6^{4}, 8^{4}$, and $10^{4}$ demonstrate that the system has three regions of different critical behaviour which are characterized by the value of $k$. The thermal cycles with steps $\delta \beta=0.001$ have been performed in a large interval of the self-intersection coupling constant $k$ from zero to four and we see that the phase structure essentially depends on $k$.

For the system with the self-intersection coupling constant $k>k_{c}^{\prime}=1 / 2$ we observe the second-order phase transition at temperature $\beta_{c} \simeq 1.75$ (see Figures $1,2)$. figures 1,2 show the energy per edge as a function of $\beta$ in Monte Carlo simulations. The energy is a continuous function of temperature for large values of the self-intersection coupling constant $k$. The critical temperature does not actually depend on $k$. The small hysteresis loop still seen on the figures is a remnant of the loop which shrinks when we change $\delta \beta$ from 0.1 to 0.001 and pass to large lattices, this is indicative for the second-order phase transition. The behaviour of the simple edges, self-intersections and plaquette averages are shown respectively on figures 3,4 and 5. From the figures 3 and 4 one can conclude that only at high temperature $\beta \leq 0.5$ the energy is concentrated on the self-intersections and that at the phase transition point the amount of energy on the self-intersections is negligible. The behaviour of the plaquette average on figure. 5 shows that the area of the random surfaces scales at the critical point and signals that the string tension is generated by quantum fluctuations, as it is expected theoretically (see (2)). The Creutz ratio [13] will be presented in a separate place.

For smaller values of $k$ the system undergoes a first order phase transition at temeprature $\beta_{c} \geq 2$ (see figures 6,7 and 8 ). The energy is discontinuous function of temperature, see figure 6 . The critical temperature depends on $k$ and is equal 


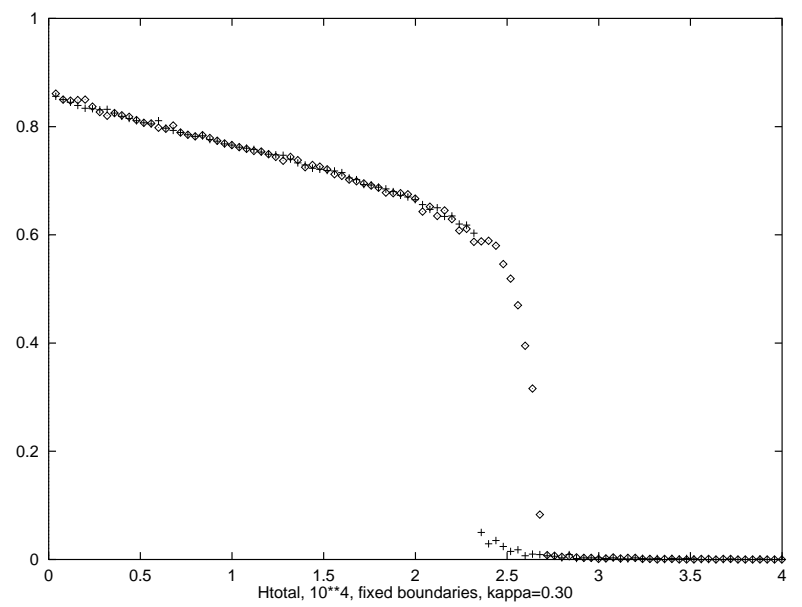

Figure 6: Hysteresis loop for total energy on edge, $k=0.3$

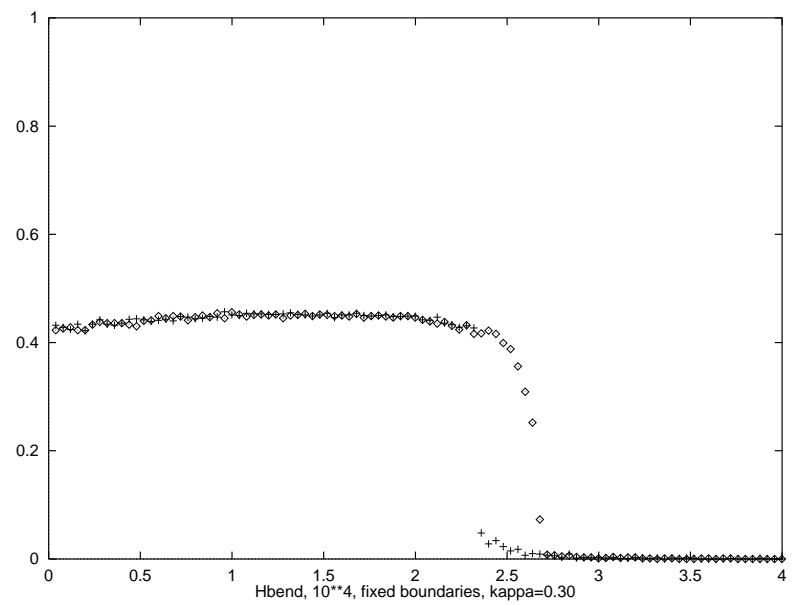

Figure 7: Hysteresis loop for simple edges, $k=0.3$

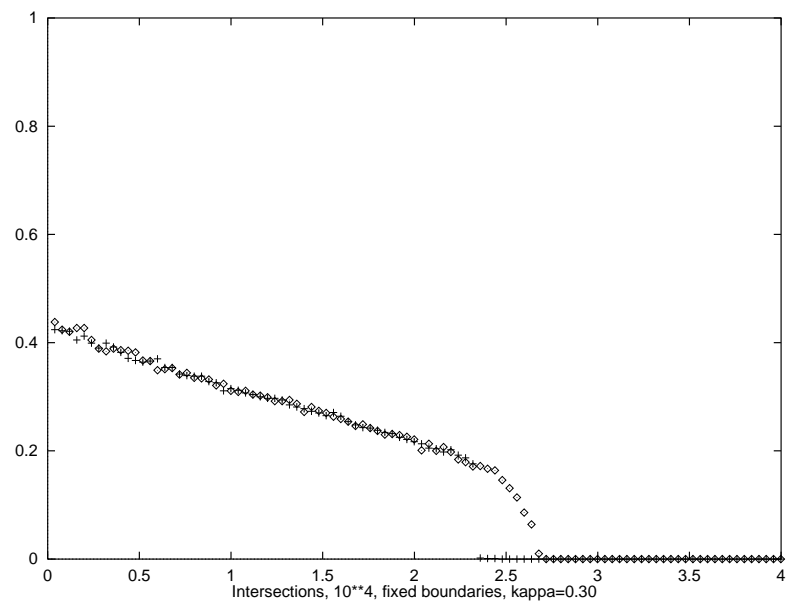

Figure 8: Hysteresis loop for self-intersection edges, $k=0.3$ 


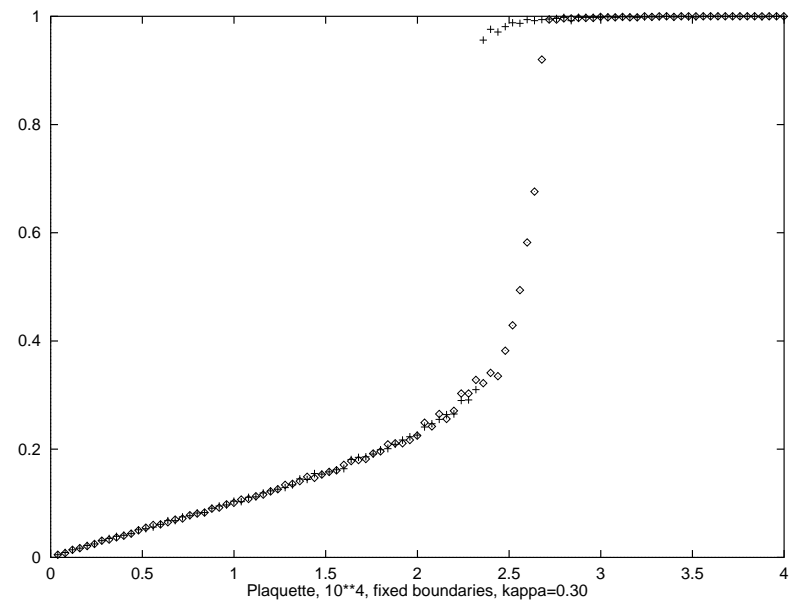

Figure 9: Hysteresis loop for plaquette, $k=0.3$

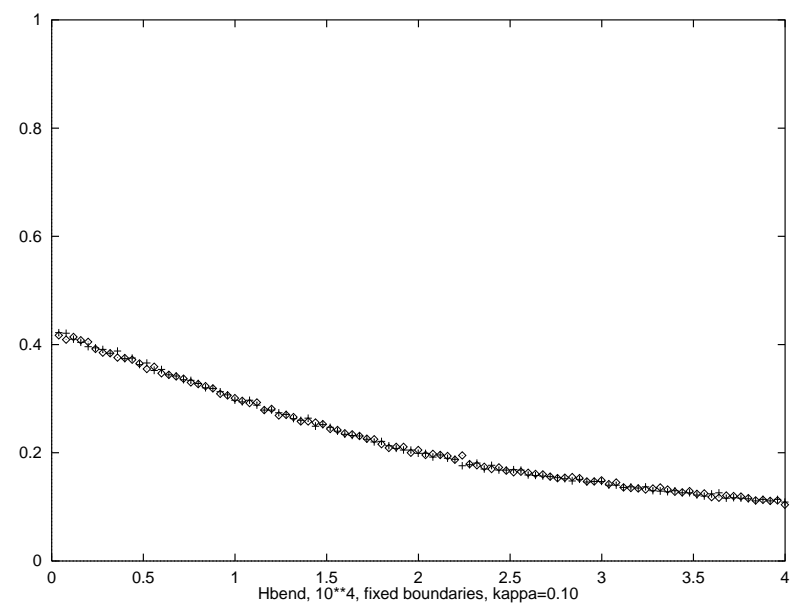

Figure 10: Simple edges, $k=0.1$

to $\beta_{c} \simeq 2.5$ for $k=0.3$. At the phase transition point the amount of energy on self-intersections considerably increases ( compare figures 2, 3, 4 and figures $6,7,8$ ) and is comparable with the energy on simple edges ( see figures 7 and 8). Thus the passage from the second-order phase transition to the first-order one is caused by the condensation of self-intersections. The reason why $k_{c}^{\prime}=1 / 2$ is a critical value for the self-intersection coupling constant is that at this value the self-intersection energy can be equivalently considered as being a sum of simple edges. The selfintersection dissolves into a number of simple edges and there is no suppression any more of self-intersections compared with the simple edges. The plaquette average shows strong discontinuity, see Fig.9, and the area does not scale in this case.

Finally, for $k<k_{c}^{\prime \prime}=1 / 6$ the system exhibits a smooth crossover, see Fig.10, and surfaces "evaporate" at all temperatures. 


\section{Conclusion}

In conclusion we would like to stress that for the system with the large self-intersection coupling constant $k$ we observe the second-order phase transition at temperature $\beta_{c} \simeq 1.75$. The string tension is generated by quantum fluctuations as it was expected theoretically [9]. This result suggests the existence of a noncritical string theory in four dimensions.

\section{References}

[1] G.K.Savvidy and F.J.Wegner. Nucl.Phys.B413(1994)605.

[2] G.K. Savvidy and K.G. Savvidy. Phys.Lett. B324 (1994) 72

[3] F.J. Wegner, J. Math. Phys.12 (1971) 2259

[4] M.Creutz, L. Jacobs and C. Rebbi Phys.Rev.Lett. 42 (1979) 1390

[5] C.Fan and F.Y.Wu. Phys.Rev.179 (1969)560

F.Y.Wu. Phys.Rev.183 (1969) 604

W. Selke, Physics Reports 170 (1988) 213

M.E. Fisher and W.Selke, Phys. Rev. Lett. 44(1980) 1502

E.I. Dinaburg and Ya.G. Sinai, Comm.Math.Phys. 98 (1985) 119

D.P. Landau, K. Binder, Phys. Rev. B 31 (1985) 5946

A.Cappi, P.Colangelo, G.Gonella and A.Maritan Nucl.Phys. B370 (1992) 659

[6] A.Maritan and C.Omero. Phys.Lett. B109 (1982) 51

T.Sterling and J.Greensite. Phys.Lett. B121 (1983) 345

B.Durhuus,J.Fröhlich and T.Jonsson. Nucl.Phys.B225 (1983) 183

T.Hofsäss and H.Kleinert. Phys.Lett. A102 (1984) 420

M.Karowski and H.J.Thun. Phys.Rev.Lett. 54 (1985) 2556

F.David. Europhys.Lett. 9 (1989) 575

[7] R.V. Ambartzumian, G.K. Savvidy , K.G. Savvidy and G.S. Sukiasian. Phys. Lett. B275 (1992) 99

[8] G.K. Savvidy and K.G. Savvidy. Int. J. Mod. Phys. A8 (1993) 3993.

[9] G.K. Savvidy and K.G. Savvidy. Mod.Phys.Lett. A8 (1993) 2963.

[10] B.Durhuus and T.Jonsson. Phys.Lett. B297 (1992) 271

[11] C.F.Baillie and D.A.Johnston. Phys.Rev D 45 (1992) 3326

[12] R.Balian, J.M.Drouffe and C.Itzykson. Phys.Rev. D10 (1974) 3376;

Phys.Rev. D11 (1975) 2098, 2104

[13] L.Kadanoff et al., Rev.Mod.Phys. 39 (1967) 395

K.G.Wilson and J.Kogut, Phys.Rev. 12C (1974) 75

M.Creutz, Quarks, gluons and lattices. (Cambridge University Press,Cambridge 
1983)

A.Polyakov. Gauge fields and String. (Harwood Academic Publishers, 1987) J.Zinn-Justin. Quantum field theory and critical phenomena. (Oxford University Press, New York 1989)

[14] G. Bhanot and M.Creutz Phys.Rev. D21 (1980) 2892

[15] G.K. Savvidy and K.G. Savvidy. Phys.Lett. B337 (1994) 333 Mod.Phys.Lett. A11 (1996) 1379.

[16] G.K. Savvidy, K.G. Savvidy and P.G. Savvidy. Phys.Lett. A221 (1996) 233

[17] G.K.Bathas, E.Floratos, G.K.Savvidy and K.G.Savvidy. Mod.Phys.Lett. A10 (1995) 2695

[18] R. Pietig and F.J. Wegner. Nucl.Phys. B466 (1996) 513

[19] M.Baig, D.Espriu, D.Johnson and R.K.P.C.Malmini, J.Phys. A30 (1997) 407; hep-lat/9703008

[20] E.Cirillo, G.Gonnella and A.Pellizzola, cond-mat/9612001

[21] G.Bhanot, M.Creutz and H.Neuberger Nucl.Phys.B235 (1984) 417;

M.Creutz. Ann. Phys.167 (1986) 62

[22] G.K.Savvidy and N.G.Ter-Arutyunian, On the Monte-Carlo Simulation of Physical Systems, J.Comput.Phys. 97, 566 (1991);

Preprint EPI-865(16)-86, Yerevan Jun.1986.

[23] N.Z.Akopov,G.K.Savvidy and N.G.Ter-Arutyunian, Matrix Generator of Pseudorandom Numbers, J.Comput.Phys.97, 573 (1991);

Preprint EPI-867(18)-86, Yerevan Jun.1986; 